\title{
ERRATUM
}

Diane W. Davidson - Steven C. Cook $\cdot$ Roy R. Snelling

\section{Liquid-feeding performances of ants (Formicidae): ecological and evolutionary implications}

Published online: 23 February 2005

(C) Springer-Verlag 2005

\section{Oecologia (2004) 139:255-266}

In the legend to Fig. 2 a number of symbols were wrongly described. The correct version is as follows:

Fig. 2 Dolichoderinae (yellow): filled square Dolichoderus (plesiomorphic proventriculus), filled circle Azteca (sc sclerotized cupola with occlusory tract), filled inverted triangle Forelius (sc, reflexed), open square Technomyrmex (sc, reflexed), open circle Tapinoma sessile (sc), filled diamond Dorymyrmex (sc, reflexed). Myrmicinae (blue): open circle Crematogaster, open triangle Cephalotes, open square Cataulacus, open inverted triangle Megalomyrmex, filled diamond Myrmicaria (sclerotized portal), ...

The online version of the original article can be found at http:// dx.doi.org/10.1007/s00442-004-1508-4

D. W. Davidson $(\bowtie)$

Department of Biology, University of Utah,

257 South, 1400 East, Salt Lake City,

UT 84112-0840, USA

E-mail: davidson@biology.utah.edu

Fax: + 1-801-5814668

S. C. Cook

Department of Biology, University of Utah,

Salt Lake City, UT 84112-0840, USA

R. R. Snelling

Entomology Section, Natural History Museum of Los Angeles,

900 Exposition Boulevard,

Los Angeles, CA 90007, USA 